\title{
Accretion properties of T Tauri stars in $\sigma$ Orionis ${ }^{\star}$
}

\author{
T. Gatti ${ }^{1,2}$, A. Natta ${ }^{1}$, S. Randich ${ }^{1}$, L. Testi ${ }^{1,3}$, and G. Sacco ${ }^{4}$ \\ 1 Osservatorio Astrofisico di Arcetri, INAF, Largo E. Fermi 5, 50125 Firenze, Italy \\ e-mail: natta@arcetri.astro.it \\ 2 Università di Firenze, Dipartimento di Astronomia, Largo E. Fermi 5, 50125 Firenze, Italy \\ 3 ESO, Karl-Schwarschild Strasse 2, 85748 Garching bei München, Germany \\ 4 Osservatorio Astronomico di Palermo, INAF, Piazza del Parlamento 1, 90134 Palermo, Italy
}

Received 31 October 2007 / Accepted 16 January 2008

ABSTRACT

\begin{abstract}
Accretion disks around young stars evolve in time with time scales of a few million years. We present here a study of the accretion properties of a sample of 35 stars in the $\sim 3$ million-year-old star-forming region $\sigma$ Ori. Of these, 31 are objects with evidence of disks, based on their IR excess emission. We use near-IR hydrogen recombination lines (Pay) to measure their mass accretion rate. We find that the accretion rates are significant lower in $\sigma$ Ori than in younger regions, such as $\rho$ Oph, consistently with viscous disk evolution. The He I $1.083 \mu \mathrm{m}$ line is detected (either in absorption or in emission) in $72 \%$ of the stars with disks, also providing evidence of accretion-powered activity in very low accretors, where other accretion indicators disappear.
\end{abstract}

Key words. stars: formation - accretion, accretion disks

\section{Introduction}

At the time of their birth, circumstellar disks are present around most (if not all) stars of mass lower than a few solar masses. The fraction of stars with disks decreases with time, from being close to $100 \%$ in the youngest regions to less than a few percent after 5-7 Myr (e.g., Hernández et al. 2007, and references therein). By the time a region is $10 \mathrm{Myr}$ old, all the "classical" (i.e., gas-rich, optically thick) disks have disappeared. On similar timescales, all other indications of accretion-powered activity, such as accretion and ejection of matter, disappear as well (e.g., Kenyon et al. 2005; Mohanty et al. 2005; Barrado y Navascués \& Martín 2003). These time scales are consistent to zero order with viscous disk evolution (Hartmann et al. 1998), although many other processes may play a role in disk dissipation (e.g., Hollenbach et al. 2000).

Recently, the discussion on how disks evolve has gained new momentum from the large surveys of star forming regions obtained with Spitzer, which provide measurements of the fraction of stars with disks based on well-defined, statistically significant samples. Particularly interesting is the new class of "evolved" disks, of which very few were known from ground-based photometry (Skrutskie et al. 1990). They are objects with IR excess emission weaker than typical classical T Tauri stars (CTTS); a fraction of these are so-called transitional disks, i.e., objects with no near-IR excess, but excess emission at longer wavelengths. The fraction of evolved disks is larger in older regions (Hernández et al. 2007). The weak emission of evolved disks may be the result of grain coagulation and settling (D'Alessio et al. 2006; Dullemond \& Dominik 2005). Transitional objects are likely disks where small grains have been cleared from the inner regions, and the discussion on their relevance for disk evolution (do all disks disappear from inside out?) is open.

* Based on observations collected at the European Southern Observatory, Chile. Program 078.C-0382.
Another indication of the presence of disks is given by the accretion-powered phenomena that go under the label of accretion activity (UV veiling, line emission and absorption from infalling and ouflowing gas, etc.). From them, it is possible to measure the mass accretion rate from the disk onto the central object, a crucial quantity for constraining the physical properties of accretion disks. Recently, Sicilia-Aguilar et al. (2006b) measured the mass accretion rate in a number of stars of different age from Taurus, $\rho$ Oph, Cha I, TW Hya and Tr 37 and found that $\dot{M}_{\text {acc }}$ decreases steadily with time, roughly as $t^{-1.5}$. Such a trend is consistent with the expectations of viscous disk evolution (Hartmann et al. 1998).

The sample used by Sicilia-Aguilar et al. (2006b) is based on individual stellar ages and includes only a few stars in each region, with the exception of $\operatorname{Tr} 37$, which contributes more than half of the total sample. The mass range of the stars with measured accretion rate in $\operatorname{Tr} 37$ (which has a distance of $\sim 900 \mathrm{pc}$ ) is shifted toward higher mass than the other, closer regions. We think that it is important to measure mass accretion rates in large numbers of stars in different regions over a large range of average ages. This is because there is evidence that, in addition to time dependence, other parameters, not identified so far, are likely to affect disk evolution, as shown by the Muzerolle et al. (2003) and Natta et al. (2006) studies of accretion in Taurus and Ophiuchus. These authors have shown that, first, the accretion rate depends on the mass of the central object and that, at the same time, there is a large range of disk and accretion properties even for stars of similar mass and age in the same star forming region. Moreover, there is evidence that the disk lifetime is shorter for more massive stars (Hillenbrand et al. 1998; Carpenter et al. 2006; Lada et al. 2006; Hernández et al. 2007; Dahm \& Hillenbrand 2007).

We are interested in extending the studies of the accretion properties of young stars to older regions, to see how they change with time and, more specifically, if both the correlation between 
accretion rate and central mass and the large spread of accretion rates, remain unchanged in time. In this paper, we present the first results of a study of the accretion properties of CTTS in $\sigma$ Ori. The $\sigma$ Ori cluster is located at a distance of $\sim 350 \mathrm{pc}$ and has an age of $\sim 3$ Myr. It contains more than 300 stars, ranging in mass from the bright, massive multiple system $\sigma$ Ori itself (spectral type 09.5) to brown dwarfs. The $\sigma$ Ori region has been extensively studied over the last few years in the optical, X-ray, and infrared (e.g., Kenyon et al. 2005; Zapatero-Osorio et al. 2002; Franciosini et al. 2006; Oliveira et al. 2004, 2006; Caballero 2007; Caballero et al. 2007). Spitzer has recently produced a complete census of the stellar population down to the brown dwarf regime (Hernández et al. 2007). These authors find that the fraction of stars that retain classical (i.e., flared, gas-rich) disks (i.e., bona fide Class II objects) vary from about $10 \%$ for stars with mass $>2 M_{\odot}$ to about $35 \%$ for T Tauri stars (TTS) and brown dwarfs (BDs). A relatively large fraction of TTS, about $15 \%$, have evolved disks; 7 of these are candidate transitional disks. The large and well characterized sample of Class II and evolved disks in $\sigma$ Ori makes it particularly suited to study the properties of accretion and disks for an intermediate age stellar population.

We determine the accretion rate from the luminosity of the IR hydrogen recombination lines, $\mathrm{Pa} \beta$, and $\mathrm{Pa} \gamma$, in particular. The reliability of this method has been discussed by Muzerolle et al. (1998), Natta et al. (2004) and Calvet et al. (2004) and applied by Natta et al. (2006) to a complete sample of IR-selected Class II stars in the young, embedded star forming region $\rho$ Oph. Although for the optically-visible objects in $\sigma$ Ori, other methods of measuring the accretion rate could (and should) be used, we consider that the comparison between different regions is more significant if the same method is used in all cases.

We discuss the properties of our $\sigma$ Ori sample in Sect. 2, which also presents details of the observations and data reduction. The results are presented in Sect. 3. The accretion rate measurements and their implications are discussed in Sect. 4. Summary and conclusions follow in Sect. 5.

\section{Sample, observations and, data reduction}

\subsection{The sample}

Our sample contains 35 TTS with evidence of accretion and/or disks. We selected them from two different sources. The first is Oliveira et al. (2004, 2006), who detected a $K-L^{\prime}$ color excess, very likely due to a circumstellar disk, in about $25 \sigma$ Ori TTS. The other source is the high spectral resolution spectral survey of Sacco et al. (2007), who detected a total of 28 stars with broad $\mathrm{H} \alpha$ lines $\left(10 \% \mathrm{FW}>200 \mathrm{~km} \mathrm{~s}^{-1}\right)$, indicative of accretion. We imposed a limiting magnitude $J<14.5$, to achieve the required signal-to-noise. Our sample includes 18 of the 23 Oliveira et al. sources above the $J$ limit, and 22/28 of the broad $\mathrm{H} \alpha$ stars; 5 stars (\#5, \#6, \#8, \#14, and \#22 in Table 1) are in common (they have both $K-L^{\prime}$ color excess and strong $\mathrm{H} \alpha$ ), for a total of 35 objects. Sacco et al. (2007) confirm the membership of the 22 stars in their sample; we find that none are binary.

After completion of our observations, Hernández et al. (2007), published the results of their Spitzer survey of the $\sigma$ Ori region. Of our 35 objects, 32 lie in the surveyed field. Based on the shape of their spectral energy distribution (SED) in the near and mid-IR, 26 of them are classified as Class II, or classical TTS (CTTS), i.e., objects with gas-rich, flared disks extending close to the star; 4 are Class III (i.e., TTS with no evidence of disk) and 2 (\#28 and \#29) are evolved

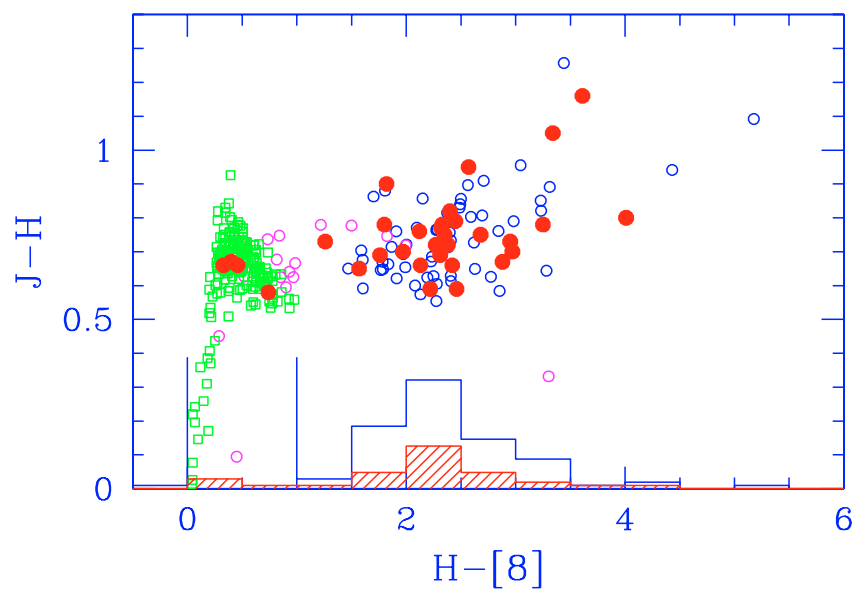

Fig. 1. $[J-H]$ versus $H-$ [8] color diagram for $\sigma$ Ori cluster members with $J<14.4$. Empty circles and squares are Class II/transitional disks and Class III objects respectively. Red filled circles show the location of our $\sigma$ Ori sample with Spitzer observations (32 objects in total). The histograms at the bottom show the distribution in $H-$ [8] of all cluster members (empty) and of our sample (dashed), respectively. All Class II disks have $H-[8]>1.5$.

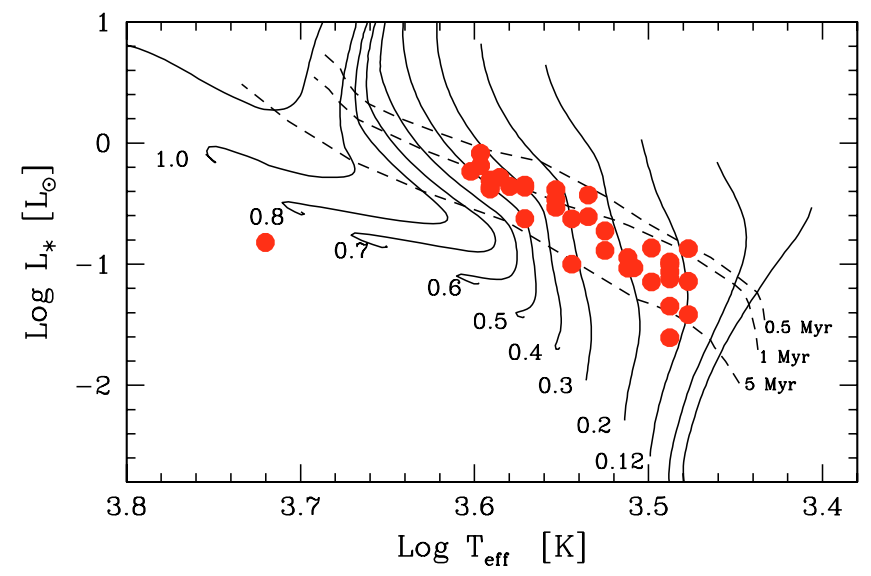

Fig. 2. Location of the $\sigma$ Ori targets on the HR diagram. Evolutionary tracks from D'Antona \& Mazzitelli (1997), labeled with the stellar mass. Isochrones for $0.5,1$ and $5 \mathrm{Myr}$ are shown by the dashed lines.

disks, i.e., objects with IR excess weaker than typical CTTS. In the following, we will analyze them together with the Class II objects and use the definition of Class II for the whole disk sample (but see Sect. 4.4). The SED classification is given in Table 1. Three stars, \#1, \#13, and \#33, are not in the fields observed by Hernández et al. (2007). We classify them as Class II based on the presence of a $K-L^{\prime}$ excess (Oliveira et al. 2004; 2006); in one case (\#1) the excess is at the $2.5 \sigma$ level, and the classification uncertain.

Within our $J$-magnitude limit $(J \leq 14.5)$, our sample of disk objects contains about $30 \%$ of the $\sigma$ Ori members with evidence of disks from the Spitzer survey. Their distribution in color and magnitude is very similar to that of the parental population (see Fig. 1), so that we consider our total sample of 31 Class II objects to be representative of the whole disk population.

\subsection{Stellar parameters}

Spectroscopically determined spectral types exist for a small fraction of $\sigma$ Ori objects only (e.g., Franciosini et al. 2006, and references therein). To ensure homogeneity, we determine 
Table 1. Stellar parameters and observed properties.

\begin{tabular}{|c|c|c|c|c|c|c|c|c|c|c|c|c|c|c|}
\hline $\begin{array}{c}\text { Obj } \\
\#\end{array}$ & $\begin{array}{c}\text { Spitz. } \\
\#\end{array}$ & $\begin{array}{c}\text { RA } \\
(2000)\end{array}$ & $\begin{array}{c}\text { Dec } \\
(2000)\end{array}$ & $\begin{array}{c}J \\
(\mathrm{mag})\end{array}$ & ST & $\mathrm{Cl}$. & $\begin{array}{c}L_{*} \\
\left(L_{\odot}\right)\end{array}$ & $\begin{array}{c}M_{*} \\
\left(M_{\odot}\right)\end{array}$ & $\begin{array}{l}T_{\text {eff }} \\
(\mathrm{K})\end{array}$ & $\begin{array}{l}\mathrm{Pa} \beta \\
(\AA)\end{array}$ & $\begin{array}{l}\mathrm{Pa} \gamma \\
(\AA)\end{array}$ & $\begin{array}{l}\mathrm{HeI} \\
(\AA)\end{array}$ & $\begin{array}{l}L_{\text {acc }} \\
\left(L_{\odot}\right)\end{array}$ & $\begin{array}{c}\dot{M}_{\mathrm{acc}} \\
\left(M_{\odot} / \mathrm{yr}\right)\end{array}$ \\
\hline 1 & - & $5: 37: 56.1$ & $-2: 09: 26.7$ & 13.90 & M4.5 & II & 0.04 & 0.16 & 3075 & $<0.5$ & $<0.6$ & - & $<-3.5$ & $<-10.4$ \\
\hline 2 & 283 & $5: 37: 58.4$ & $-2: 41: 26.2$ & 13.28 & M5.0 & III & 0.07 & 0.12 & 3000 & $<0.5$ & $<0.4$ & - & $<-3.4$ & $<-10.0$ \\
\hline 3 & 451 & $5: 38: 18.9$ & $-2: 51: 38.8$ & 12.83 & M3.0 & II & 0.12 & 0.25 & 3350 & $<0.5$ & $2.0 \pm 0.4$ & $1.7 \pm 0.3$ & -2.2 & -9.1 \\
\hline 4 & 462 & $5: 38: 20.5$ & $-2: 34: 09.0$ & 12.65 & M4.0 & II & 0.13 & 0.16 & 3150 & $<0.4$ & $<0.3$ & - & $<-3.2$ & $<-9.8$ \\
\hline 5 & 518 & $5: 38: 27.3$ & $-2: 45: 09.7$ & 11.95 & K8.0 & II & 0.42 & 0.5 & 3900 & $9.4 \pm 0.5$ & $6.7 \pm 0.6$ & $7.3 \pm 0.5$ & -1.0 & -8.1 \\
\hline 6 & 520 & $5: 38: 27.5$ & $-2: 35: 04.2$ & 12.83 & M3.5 & II & 0.12 & 0.2 & 3250 & $<0.6$ & $0.8^{c}$ & - & -2.8 & -9.6 \\
\hline 7 & 562 & $5: 38: 31.4$ & $-2: 36: 33.8$ & 12.17 & M3.0 & II & 0.19 & 0.2 & 3350 & $4.9 \pm 0.4$ & $3.6 \pm 0.3$ & $4.7 \pm 0.3$ & -1.5 & -8.2 \\
\hline 8 & 563 & $5: 38: 31.6$ & $-2: 35: 14.9$ & 11.52 & M2.0 & II & 0.23 & 0.3 & 3500 & $<0.5$ & $<0.5$ & $1.8 \pm 0.5$ & $<-2.3$ & $<-9.2$ \\
\hline 9 & 592 & $5: 38: 34.3$ & $-2: 35: 00.1$ & 11.22 & K0.0 & III & 0.16 & 0.8 & 5250 & $<0.4$ & $<0.4$ & - & $<-2.3$ & $<-10.0$ \\
\hline 10 & 598 & 5:38:34.6 & $-2: 41: 08.8$ & 13.10 & M4.5 & II & 0.11 & 0.14 & 3075 & $<0.6$ & $<0.7$ & $-0.8^{c}$ & $<-3.0$ & $<-9.6$ \\
\hline 11 & 611 & $5: 38: 35.5$ & $-2: 31: 51.7$ & 11.30 & K7.0 & III & 0.57 & 0.5 & 4000 & $<0.7$ & $<0.7$ & - & $<-2.0$ & $<-9.0$ \\
\hline 12 & 646 & $5: 38: 39.0$ & $-2: 45: 32.2$ & 12.91 & M3.5 & II & 0.10 & 0.2 & 3225 & $<0.4$ & $0.8 \pm 0.3$ & $-1.8 \pm 0.4$ & -2.8 & -9.6 \\
\hline 13 & - & $5: 38: 39.8$ & $-2: 56: 46.2$ & 11.43 & M0.5 & II & 0.45 & 0.4 & 3725 & $<0.7$ & $<0.4$ & $2.8 \pm 0.3$ & $<-2.4$ & $<-9.3$ \\
\hline 14 & 662 & $5: 38: 40.3$ & $-2: 30: 18.5$ & 11.51 & M0.5 & II & 0.44 & 0.4 & 3725 & $<0.7$ & $0.7^{c}$ & $-0.7^{c}$ & -2.1 & -9.0 \\
\hline 15 & 669 & $5: 38: 41.3$ & $-2: 37: 22.6$ & 11.46 & M0.0 & III & 0.44 & 0.45 & 3800 & $<0.7$ & $<0.4$ & $0.7^{c}$ & $<-2.4$ & $<-9.4$ \\
\hline 16 & 682 & $5: 38: 42.3$ & $-2: 37: 14.7$ & 11.77 & M1.5 & II & 0.35 & 0.3 & 3575 & $<0.4$ & $<0.5$ & $-1.2 \pm 0.4$ & $<-2.5$ & $<-9.3$ \\
\hline 17 & 694 & $5: 38: 43.9$ & $-2: 37: 06.8$ & 12.84 & M4.5 & II & 0.1 & 0.14 & 3075 & $<0.4$ & $<0.4$ & $1.0 \pm 0.3$ & $<-3.2$ & $<-9.8$ \\
\hline 18 & 697 & $5: 38: 44.2$ & $-2: 40: 19.7$ & 11.36 & K7.5 & II & 0.63 & 0.45 & 3950 & $<0.5$ & $<0.3$ & $2.1 \pm 0.3$ & $<-2.5$ & $<-9.5$ \\
\hline 19 & 710 & $5: 38: 45.4$ & $-2: 41: 59.4$ & 11.99 & M2.5 & II & 0.25 & 0.25 & 3425 & $<0.6$ & $1.3 \pm 0.3$ & $0.3^{d}$ & -2.0 & -8.8 \\
\hline 20 & 723 & $5: 38: 47.2$ & $-2: 34: 36.8$ & 12.56 & M3.5 & II & 0.09 & 0.2 & 3250 & $3.4 \pm 0.5$ & $2.1 \pm 0.4$ & - & -2.0 & -8.9 \\
\hline 21 & 726 & $5: 38: 47.5$ & $-2: 35: 25.2$ & 11.74 & M1.5 & II & 0.42 & 0.3 & 3575 & $1.1 \pm 0.3$ & $1.4 \pm 0.3$ & $0.0^{e}$ & -1.8 & -8.6 \\
\hline 22 & 733 & $5: 38: 47.9$ & $-2: 37: 19.2$ & 12.02 & M2.5 & II & 0.36 & 0.25 & 3425 & $<0.4$ & $1.3 \pm 0.3$ & $0.6^{c}$ & -2.0 & -8.7 \\
\hline 23 & 736 & $5: 38: 48.0$ & $-2: 27: 14.2$ & 10.16 & K8.0 & II & 0.83 & 0.45 & 3950 & $<0.6$ & $<0.5$ & $-1.5 \pm 0.4$ & $<-1.6$ & $<-8.5$ \\
\hline 24 & 750 & $5: 38: 49.3$ & $-2: 23: 57.6$ & 14.36 & M4.5 & II & 0.03 & 0.16 & 3075 & $<1.2$ & $<1.2$ & - & $<-3.4$ & $<-10.3$ \\
\hline 25 & 762 & $5: 38: 50.6$ & $-2: 42: 42.9$ & 13.84 & M5.0 & II & 0.04 & 0.12 & 3000 & $<0.5$ & $<0.7$ & $2.9 \pm 0.4$ & $<-3.4$ & $<-10.1$ \\
\hline 26 & 774 & $5: 38: 52.0$ & $-2: 46: 43.7$ & 11.52 & K8.0 & II & 0.44 & 0.5 & 3900 & $<0.5$ & $1.6 \pm 0.5$ & $5.2 \pm 0.5$ & -1.6 & -8.7 \\
\hline 27 & 871 & 5:39:04.6 & $-2: 41: 49.4$ & 13.96 & M2 & II & 0.1 & 0.3 & 3500 & $<1.2$ & $<1.0$ & $8.5 \pm 1.0$ & $<-3.2$ & $<-10.3$ \\
\hline 28 & 897 & 5:39:07.6 & $-2: 32: 39.1$ & 11.30 & K8.0 & $\mathrm{II}^{a}$ & 0.6 & 0.5 & 3900 & $<0.7$ & $<0.6$ & $1.2^{c}$ & $<-2.1$ & $<-9.1$ \\
\hline 29 & 908 & $5: 39: 08.8$ & $-2: 31: 11.5$ & 13.07 & M4.5 & $\mathrm{II}^{b}$ & 0.07 & 0.16 & 3075 & $1.3 \pm 0.4$ & $0.8 \pm 0.3$ & $-1.3 \pm 0.3$ & -2.9 & -9.6 \\
\hline 30 & 927 & $5: 39: 11.5$ & $-2: 31: 06.6$ & 11.99 & M0.5 & II & 0.24 & 0.5 & 3725 & $<0.8$ & $<0.7$ & - & $<-2.4$ & $<-9.5$ \\
\hline 31 & 984 & $5: 39: 18.8$ & $-2: 30: 53.1$ & 11.40 & K9.0 & II & 0.52 & 0.45 & 3850 & $<0.6$ & $<0.4$ & $-1.1 \pm 0.3$ & $<-2.4$ & $<-9.4$ \\
\hline 32 & 1152 & $5: 39: 39.4$ & $-2: 17: 04.5$ & 11.67 & M1.5 & II & 0.29 & 0.35 & 3575 & $<0.6$ & $<0.5$ & $-2.4 \pm 0.5$ & $<-2.4$ & $<-9.3$ \\
\hline 33 & - & $5: 39: 41.0$ & $-2: 16: 24.4$ & 12.91 & M4.5 & II & 0.10 & 0.14 & 3075 & $<0.4$ & $<0.4$ & - & $<-3.2$ & $<-9.8$ \\
\hline 34 & 1230 & $5: 39: 49.4$ & $-2: 23: 45.9$ & 13.44 & M4.0 & II & 0.07 & 0.18 & 3150 & $<0.8$ & $<0.8$ & $0.8^{c}$ & $<-3.1$ & $<-9.9$ \\
\hline 35 & 1248 & $5: 39: 51.7$ & $-2: 22: 47.2$ & 12.60 & M5.0 & II & 0.13 & 0.12 & 3000 & $<0.5$ & $<0.6$ & - & $<-2.8$ & $<-9.3$ \\
\hline
\end{tabular}

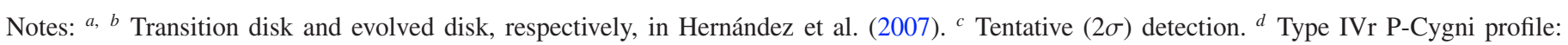
$E W(\mathrm{em})=1.2 \pm 0.3, E W(\mathrm{abs})=-0.9 \pm 0.3 .{ }^{e}$ Tentative type IVr P-Cygni profile $(0.6,-0.6)$.

the effective temperature of each object from its visual photometry, adopting the relation between color indexes, $T_{\text {eff }}$ and spectral types of Bessell (1991) for M dwarf stars, and from Bessell (1979), and Bessell \& Brett (1988) for stars of earlier spectral type. We assume negligible extinction in all bands (Brown et al. 1994; Béjar et al. 1999), and compute $T_{\text {eff }}$ independently from the three color indexes $(V-R),(V-I),(R-I)$. The optical photometry is from Sherry et al. (2004), Zapatero-Osorio et al. (2002), Kenyon et al. (2005), Béjar et al. (2001), and Wolk (1996). The differences between the different determinations of $T_{\text {eff }}$ are generally less than $\pm 150 \mathrm{~K}$, and often much smaller. The adopted values of $T_{\text {eff }}$ and spectral types are given in Table 1. The comparison with the spectroscopic determinations of spectral types of Zapatero-Osorio et al. (2002) for the 8 objects in common shows differences of $1 / 2$ to 1 subclass.

We computed stellar luminosities from the I magnitude, using the bolometric correction appropriate for the spectral type (Kenyon \& Hartmann 1995), no extinction and a distance $D=$ $350 \mathrm{pc}$. The location of the stars on the HR diagram is shown in Fig. 2, together with the evolutionary tracks of D'Antona \& Mazzitelli (DM97, 1997). The distribution of the stars is consistent with a typical age of 3 Myr (Oliveira et al. 2004), with the exception of \#9, a Class III object with color indexes consistent with an early K spectral type, but with very low luminosity.

The stellar masses range from $\sim 0.5$ to $\sim 0.1 M_{\odot}$. Note that the exact values of $M_{*}$ depend on the adopted evolutionary tracks, especially around $0.5-1 M_{\odot}$. We use DM97 for homogeneity with the analysis of Natta et al. (2006) of the mass accretion rates in $\rho$ Oph. The lower mass limit is determined by our selection criterion that $J<14.5 \mathrm{mag}$; the lack of more massive stars reflects the strong decrease of the fraction of Class II objects for increasing $J$ magnitude observed by Hernández et al. (2007). This trend, seen in other star forming regions (e.g., Hillenbrand et al. 1998; Carpenter et al. 2006; Lada et al. 2006; Hernández et al. 2007; Dahm \& Hillenbrand 2007), is particularly strong in $\sigma$ Ori, where only $4(7 \%)$ stars brighter than $J=11 \mathrm{mag}$ are classified as Class II. One of them is included in our sample (\#23), and has an estimated spectral type K8 and mass $\sim 0.5 M_{\odot}$. The fact that no higher-mass member has an associated Class II disk is confirmed by Caballero (2007): of the 18 very bright stars (spectral type G0 and earlier) in the $\sigma$ Ori cluster studied by him and included in the list of cluster members by Hernández et al. (2007), 3 have transitional or debris disks, and all the others are Class III. 


\subsection{Observations}

We have obtained near-infrared spectra in the $J$ band for all our targets using SOFI near-infrared camera and spectrograph at the ESO-NTT telescope. The observations of $\sigma$ Ori were carried out in visitor mode on December 1-4, 2006. We used the 0.6 arcsec slit and the Blue low resolution grism, resulting in a spectral resolution $\lambda / \Delta \lambda \sim 1000$ and a spectral coverage from $\sim 0.95$ to $\sim 1.64 \mu \mathrm{m}$. Integration time was $40 \mathrm{~min}$ for all the targets; standard calibrations (flats and lamps) and telluric standards spectra were obtained for each observation. Wavelength calibration was performed using the lamp observations.

The mean seeing during the three nights was 0.7 arcsec, with many hours below 0.5 arcsec. All target objects and telluric standards have been observed at airmass values $z<2$. With the goal of observing as many objects as possible, we observed the targets in pairs, choosing stars with angular separations that allow a 20 arcsec nodding and a 6 arcsec jitter with the 4.71 arcmin slit, and orienting the slit at appropriate position angles. In total, we have observed 24 targets in this way, while 11 have been observed individually.

The spectra have been reduced using standard procedures in IRAF. In this paper, we are interested in the three lines indicative of accretion powered activity that fall in the $J$ band, namely the HeI line at $1.083 \mu \mathrm{m}$, and the two hydrogen recombination lines, $\mathrm{Pa} \gamma$ at $1.094 \mu \mathrm{m}$ and $\mathrm{Pa} \beta$ at $1.282 \mu \mathrm{m}$; the portions of the spectra that contain these lines are shown in Figs. 3 and 4. Their equivalent widths are given in Table 1 (positive values for emission lines). Upper limits to $\mathrm{Pa} \beta$ and $\mathrm{Pa} \gamma$ assume that the lines are in emission; they have been estimated from the peak-to-peak noise in the spectral region of the lines, under the assumption that the lines are not resolved. Since the He line can be either in emission, or in absorption, or both, we do not explicitly give upper limits in Table 1, but they are similar to those of the adjacent Pay line.

\section{Results}

The presence of the hydrogen lines in emission and of the He I line, often with P-Cygni profiles clearly seen in high resolution spectra, (Edwards et al. 2006) is evidence of accretion related activity. In fact, inspection of Table 1 shows that none of the three lines is detected in Class III stars, with the possible exception of \#15, where we have a tentative detection of He I in emission. Of the 31 Class II, we detect at least one of the three lines in 26 cases. The hydrogen lines are detected in emission in 12 Class II stars, but we detect both of them only in 5 stars; in 7 cases, only Pa $\gamma$ is clearly seen. The hydrogen line detection is confirmed in 10/12 cases by the presence of the He I line, either in absorption or in emission.

Our spectral resolution $\left(\sim 300 \mathrm{~km} \mathrm{~s}^{-1}\right)$ is too low to resolve the lines. However, some lines appear to be marginally resolved. It is possible that weak lines look broad due to noise spikes in the adjacent continuum, as we think is the case of Pa $\gamma$ in object \#3, which has an unrealistic FWHM of $950 \mathrm{~km} \mathrm{~s}^{-1}$; we did not try to correct for this effect the equivalent width in Table 1, which is hence very likely overestimated by a factor of $\sim 2$. In two stars (\#19 and \#21), the He I line has an inverse P-Cygni profile, with redshifted absorption; the line is strong in \#19, but barely detected in \#21. Table 1 gives the total equivalent width of the line, while the values of the absorption and emission components are given in the notes to the table.

We were surprised by the number of objects where Pa $\gamma$ is detected, while $\operatorname{Pa} \beta$ is not. The ratio of the $\operatorname{Pa} \gamma$ to $\operatorname{Pa} \beta$ fluxes has been measured recently for a group of CTTS in Taurus by Bary \& Matt (2007), who find a tight correlation with a ratio $0.86 \pm 0.10$. Magnetospheric accretion models (Muzerolle et al. 2001) predict ratios $\sim 0.7-0.9$ for $\dot{M}_{\text {acc }} \gtrsim 10^{-8} M_{\odot} / \mathrm{yr}$, increasing to $\sim 1.2-1.4$ at $\dot{M}_{\text {acc }} \sim 10^{-9} M_{\odot} / \mathrm{yr}$ (Muzerolle, private communication). Considering that for TTS the ratio of the continuum at the two line wavelengths is $\sim 1.2-1.4$, we expect in any case comparable equivalent widths, and, in fact, this is the case in the five $\sigma$ Ori objects where both lines are detected. All stars with $\mathrm{Pa} \gamma$, but no $\mathrm{Pa} \beta$ have relatively weak $\mathrm{Pa} \gamma$; however, in several cases we should have detected $\operatorname{Pa} \beta$ if the equivalent widths were similar and the lines unresolved. We have checked the observational and data reduction procedures, and found no obvious explanation for the absence of $\mathrm{Pa} \beta$. It is possible that the upper limits to $\mathrm{Pa} \beta$, derived assuming that the line is not resolved, are in fact underestimated, and, indeed, some objects with very low limits to the ratio of the $\mathrm{Pa} \beta / \mathrm{Pa} \gamma$ equivalent width (e.g., \#3, \#22, \#26) have broad Pa $\gamma$ (see Fig. 3). Also, in some cases $\mathrm{Pa} \beta$ may have a P-Cygni profile with deeper absorption than Pa $\gamma$, and this could reduce the ratio of the unresolved equivalent widths. This kind of profile, however, is quite rare in Taurus TTS (e.g., Edwards et al. 2006; Folha \& Emerson 2001). Note that the two lines $\mathrm{Pa} \gamma$ and $\mathrm{Pa} \beta$ are observed simultaneously, so that variability cannot affect their ratio.

The HeI $1.083 \mu \mathrm{m}$ line is seen in $23 / 31$ Class II stars, in absorption (8 cases), in emission (14 cases), or with a P-Cygni profile ( 2 cases). This variety of profiles is known to occur in TTS (Edwards et al. 2006), and will be discussed in Sect. 4.2.

\section{Discussion}

\subsection{Accretion luminosities and mass accretion rates}

We use the hydrogen recombination lines to measure the accretion luminosity $L_{\text {acc }}$, following the procedure described in detail by Natta et al. (2006). First, we compute line fluxes from the equivalent widths and the flux of the continuum near each line, calibrated with the 2 MASS value of the $J$ magnitude and $A_{J}=0$. We apply a correction of a factor 1.3 to the continuum at $1.09 \mu \mathrm{m}$, to account for the typical slope of the $J$-band continuum in late $\mathrm{K}-\mathrm{M}$ stars.

Line luminosities are computed from the line fluxes for the adopted distance $D=350 \mathrm{pc}$. We use them to derive a measurement of the accretion luminosity $L_{\text {acc }}$ using the empirical correlation between the luminosity of the near-IR hydrogen recoombination lines and $L_{\text {acc }}$, first noticed by Muzerolle et al. (1998), and used to study the accretion properties of TTS in Ophiuchus by Natta et al. (2006). The correlation has been established quantitatively for the two lines $\mathrm{Pa} \beta$ and $\mathrm{Br} \gamma$ in a sample of TTS in Taurus for which reliable measurements of the accretion luminosity from veiling were available (Muzerolle et al. 1998; Calvet et al. 2004), and extended in the case of $\mathrm{Pa} \beta$ to the brown dwarf regime by Natta et al. (2004). We adopt here a relation between $L_{\text {acc }}$ and Pa $\gamma$ luminosity obtained from the Natta et al. (2004) correlation between $L_{\text {acc }}$ and the $\mathrm{Pa} \beta$ luminosity and the ratio of $\mathrm{Pa} \gamma$ to $\mathrm{Pa} \beta$ fluxes of $0.86 \pm 0.1$ (Bary \& Matt 2007):

$\log \frac{L_{\mathrm{acc}}}{L_{\odot}}=1.36 \times \log \frac{L(\mathrm{~Pa} \gamma)}{L_{\odot}}+4.1$.

Equation (1) does not depend on any assumption about the line origin, but only on the known empirical relation between $L_{\mathrm{acc}}$ and the $\mathrm{Pa} \beta$ luminosity and the adopted value of the $\mathrm{Pa} \gamma / \mathrm{Pa} \beta$ ratio. As discussed at the end of Sect. 3, in several of our $\sigma$ Ori objects, the observed ratio $\operatorname{Pa} \gamma / \mathrm{Pa} \beta$ is higher than the Bary and 

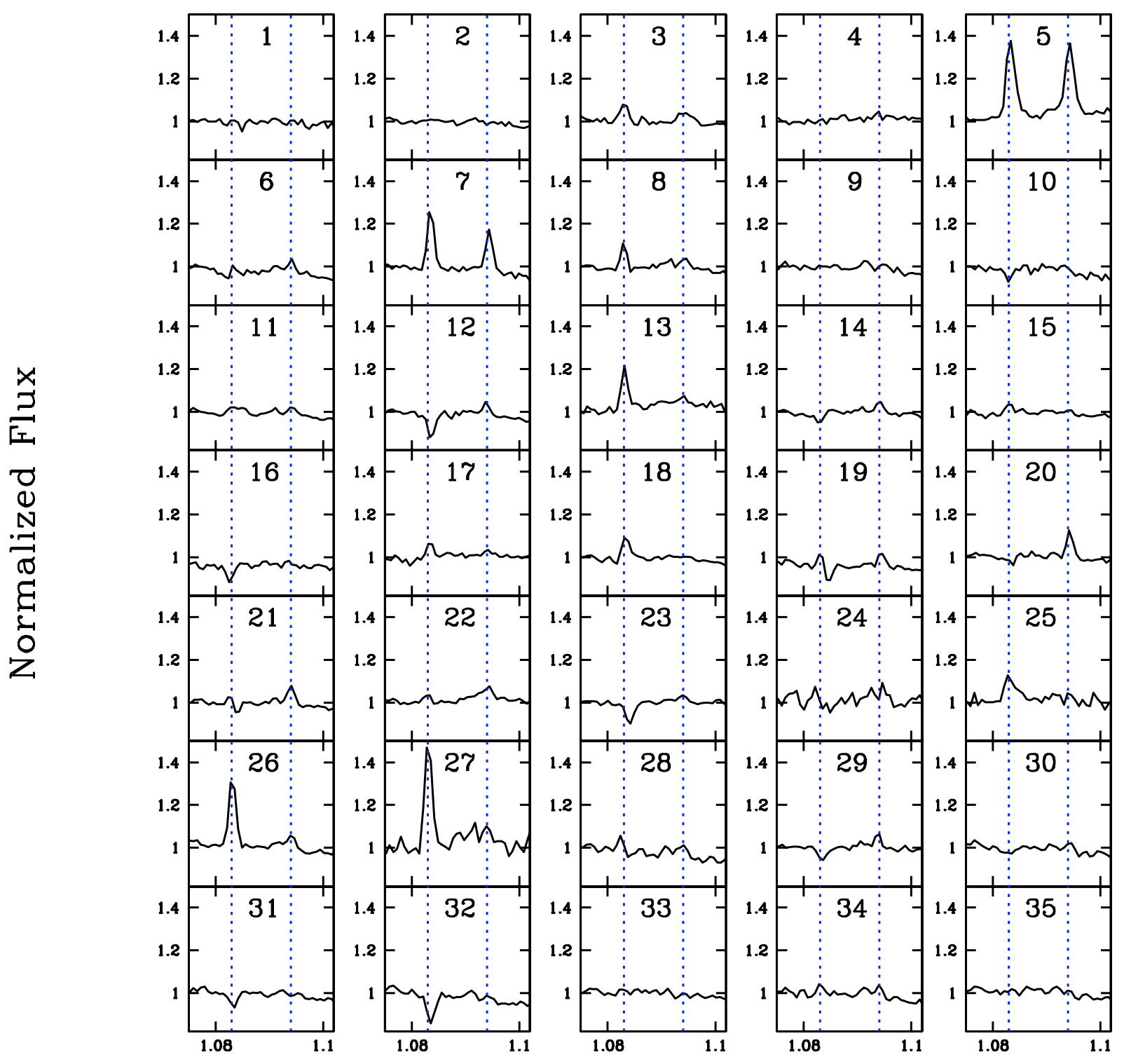

\section{Wavelength $(\mu \mathrm{m})$}

Fig. 3. Normalized observed spectra in the HeI and Pay region. The dotted lines show the wavelengths of the two lines.

Matt ratio, and the values (or upper limit) of $L_{\text {acc }}$ computed from $L(\mathrm{~Pa} \beta)$ are correspondingly lower (see Fig. 5). We decided to use, in the following, $L_{\text {acc }}$ derived from $\mathrm{Pa} \gamma$ to maximize the number of actual measurements; since $L_{\text {acc }}(\mathrm{Pa} \beta) \lesssim L_{\text {acc }}(\mathrm{Pa} \gamma)$, deriving $L_{\text {acc }}$ from $L(\mathrm{~Pa} \beta)$ would just strengthen our conclusions.

The mass accretion rate $\dot{M}_{\text {acc }}$ is then computed from $L_{\text {acc }}$ :

$\dot{M}_{\mathrm{acc}}=\frac{L_{\mathrm{acc}} R_{*}}{G M_{*}}$

where $M_{*}$ and $R_{*}$ are the stellar mass and radius, respectively.

The uncertainties on $L_{\text {acc }}$ and $\dot{M}_{\text {acc }}$ are difficult to estimate, but they are certainly not small. In addition to the measurement errors on the line equivalent widths, there are continuum calibration uncertainties since our spectra have not been photometrically calibrated. Variability in the lines is a well-known phenomenon in TTS, and snapshot estimates of their strength can differ from the long-term average values by large factors. Moreover, the relation between $L(\mathrm{~Pa} \gamma)$ and $L_{\mathrm{acc}}$ has an intrinsic, non-negligible scatter, and the determination of the stellar mass/radius depends on a number of assumptions, among them the adopted evolutionary tracks. The final uncertainties on $\dot{M}_{\text {acc }}$ can easily be of \pm 0.5 dex, as discussed, e.g., by Calvet et al. (2004) and Natta et al. (2006). In spite of all that, snapshot accretion values of a large number of stars in any given star formation region provide very significant information on the typical accretion properties of the region and on its evolutionary stage.

The results for $\sigma$ Ori are summarized in Figs. 6 and 7, top panel. In Fig. 6, we plot the accretion luminosity as a function of the stellar luminosity for all Class II objects. It shows clearly that very few stars have $L_{\text {acc }}$ larger than $0.1 L_{*}$, and that most of them have values well below this limit. This is different from the results obtained in younger star forming regions, such as Taurus and Ophiuchus (see summary in Natta et al. 2006), where a large fraction of stars has $L_{\text {acc }} / L_{*}>0.1$. 



\section{Wavelength $(\mu \mathrm{m})$}

Fig. 4. Normalized observed spectra in the $\mathrm{Pa} \beta$ region. The wavelength of $\mathrm{Pa} \beta$ is shown by the dotted line.

In Fig. 7 we show the mass accretion rate as a function of the stellar mass for the same 31 objects. The values of $\dot{M}_{\text {acc }}$ are all smaller than $\sim 10^{-8} M_{\odot} / \mathrm{yr}$, and 23/31 stars have accretion rates lower than $10^{-9} M_{\odot} /$ yr. When compared to stars with similar mass in Taurus and Ophiuchus, these are very low values, as we will discuss in Sect. 4.5.

\subsection{He line}

Edwards et al. (2006) have recently published a study of the Pay and He I profiles in a sample of 39 Taurus TTS; they found that the strength of both lines correlates with the $J$-band veiling, i.e., with the accretion luminosity. Although with a large spread, objects with low veiling have on average not only weaker but also narrower Pa $\gamma$. He I lines very often have P-Cygni profiles with blue-shifted absorption caused by outflowing gas and red-shifted absorption due to infalling material. On average, strong accretors have the He I line in emission; red-shifted and blue-shifted absorption features are present in high and low accretors, but, if unresolved, the He I equivalent width would change from strong emission in high accretors to net absorption in low accretors. In these objects, the blue-shifted absorption features due to outflowing matter, very likely a non-collimated wind, dominate the profile.

In Fig. 8 we plot the equivalent width of the $\mathrm{He}$ I line as function of the accretion luminosity $L_{\text {acc }}$ for our sample. If we consider objects with measured $L_{\text {acc }}$, we find a good correlation between the two quantities, with He I in net absorption in low accretors and in emission in high accretors, as in the Edwards et al. (2006) sample. Note that Edwards et al. use in their analysis of the He I line the "activity index", i.e., the sum of the absolute values of the emission and absorption equivalent widths, while our unresolved profiles give the net equivalent width only. The trend of decreasing emission, increasing net absorption for decreasing $L_{\text {acc }}$ is weaker if we include objects with $L_{\text {acc }}$ upper limits; as in Taurus, there are some objects with strong He I 


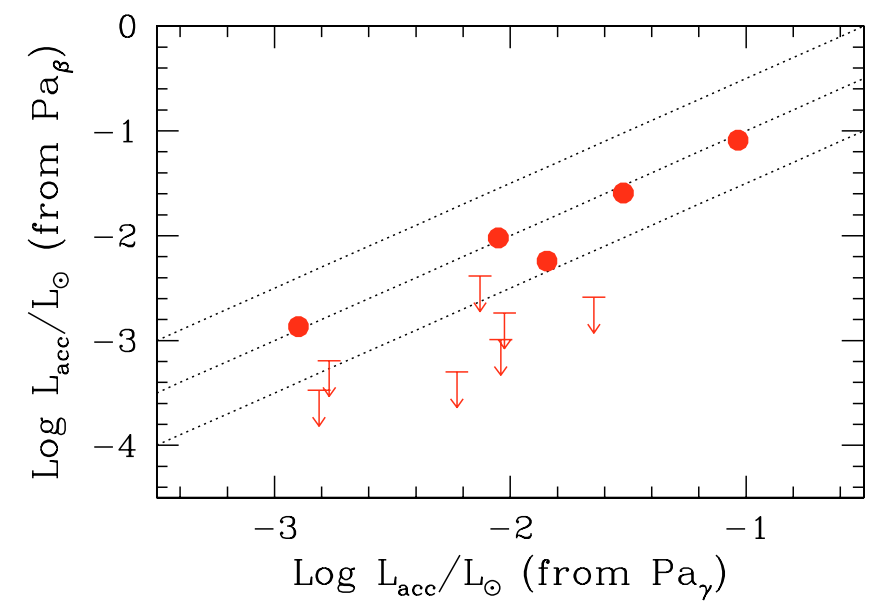

Fig. 5. Comparison of the accretion luminosity computed from Pa $\gamma$ and that computed from $\mathrm{Pa} \beta$ for all objects with $\mathrm{Pa} \gamma$ detection. Dots are actual measurements, arrow $3 \sigma$ upper limits. The dotted lines show the locus of $L_{\mathrm{acc}}(\mathrm{Pa} \gamma)=L_{\mathrm{acc}}(\mathrm{Pab})$ and the \pm 0.5 range.

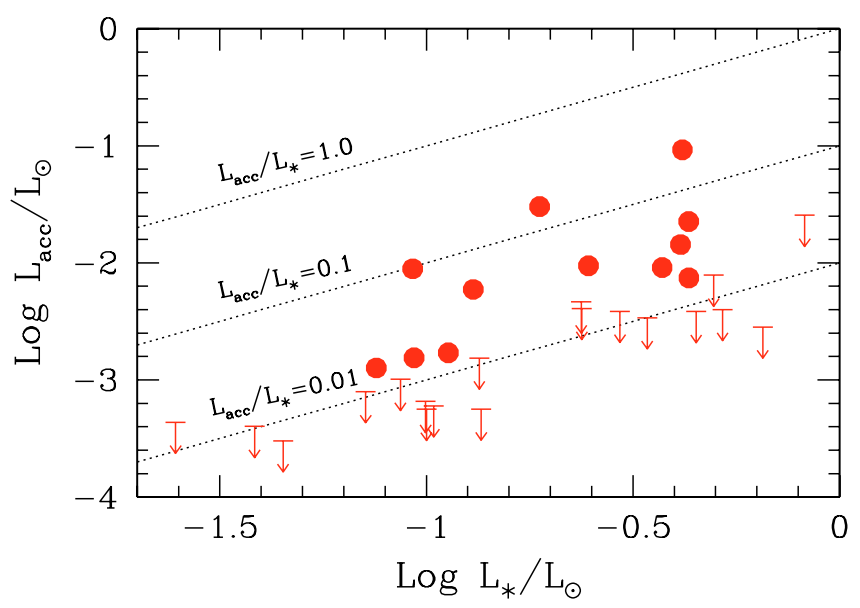

Fig. 6. Accretion luminosity as function of the stellar luminosity for the Class II objects. Dots are actual measurements, arrow $3 \sigma$ upper limits. The dotted lines show the location of the $L_{\text {acc }} / L_{*}=1.0,0.1$ and 0.01 ratios, respectively.

emission, but low $L_{\text {acc }}$ (e.g., \#27), which it would be interesting to investigate further.

The Taurus sample includes CTTS with mass accretion rates $\dot{M}_{\text {acc }}$ between $\sim 5 \times 10^{-6}$ and $5 \times 10^{-10} M_{\odot} /$ yr, with a large fraction (29/38) of objects accreting at rates higher than the highest values of the $\sigma$ Ori sample. Edwards et al. (2006) divide their sample of CTTS into four groups, according to the $1 \mu \mathrm{m}$ veiling. The median values of $\log \dot{M}_{\text {acc }}$ of the first and second group (high and medium veiling) are -5.8 and $-7 M_{\odot} / \mathrm{yr}$, while the third and fourth (low $1 \mu \mathrm{m}$ veiling, narrow and broad Pa $\gamma$, respectively) have median $-8.2 M_{\odot} / y r$. The low veiling, broad Pa $\gamma$ group has, on average, He I lines with net negative equivalent widths. The $\sigma$ Ori sample overlaps with the low veiling objects and extends it to even lower values of $\dot{M}_{\text {acc. }}$. In fact, there are no objects with the very strong He I emission lines of some Group I Taurus stars, while the fraction of objects with net He I absorption is higher than in the Edwards et al. (2006) group IV.

An interesting aspect of these results is that absorption He I lines can be detected even in objects with very low $\dot{M}_{\text {acc }}$, where direct accretion signatures (e.g., hydrogen line emission, veiling) can be hard to measure. Accretion-powered winds may be the longest lasting tracers of disk activity.

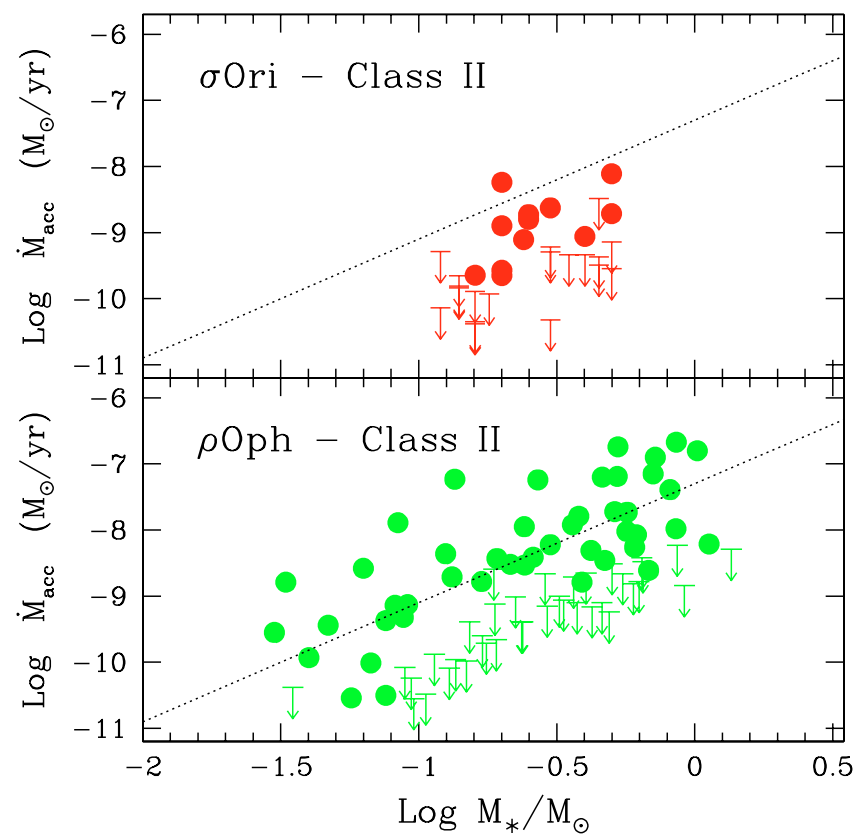

Fig. 7. Mass accretion rate as a function of $M_{*}$ for $\sigma$ Ori (top) and $\rho$ Oph (bottom; from Natta et al. 2006). Dots are actual measurements, arrows $3 \sigma$ upper limits. The dotted line (same in both panels) shows the $\log M_{\text {acc }}=-7.3+1.8 \times \log M_{\star}$ locus, which we take as a fiducial line to define "high accretors" (see text).

\subsection{Evolved disks}

The $\sigma$ Ori cluster contains a relatively high fraction of evolved disks, i.e., objects with IR excess emission lower than the median of CTTS. Some of them are classified by Hernández et al. (2007) as transitional disks, i.e., objects with a very low excess in the near-IR but a normal excess at longer wavelengths. Our sample includes two such objects. One (\#28, S897) is classified as a transitional disk; it has no $\mathrm{Pa} \beta$ or $\mathrm{Pa} \gamma$ lines above the detection limit (the corresponding mass accretion rate is $<10^{-9} M_{\odot} / \mathrm{yr}$ ), but a tentative detection of $\mathrm{He} \mathrm{I}$, and a rather broad $\mathrm{H} \alpha\left(10 \% F W=504 \mathrm{~km} \mathrm{~s}^{-1}\right.$; Sacco et al. 2007). The other object (\#29, S908) is classified as an evolved disk, but shows both $\mathrm{Pa} \beta$ and $\mathrm{Pa} \gamma$ in emission and the He I line in absorption. Also for this object, Sacco et al. (2007) measure a broad $\mathrm{H} \alpha$, with a $10 \% F W=401 \mathrm{~km} \mathrm{~s}^{-1}$. Its accretion rate is similar to that of the Class II $\sigma$ Ori stars.

It is possible that, in these objects (\#29 in particular), the low IR excess is due to the disk orientation on the plane of the sky. However, some transitional disks are known to have relatively large $\dot{M}_{\text {acc }}$ (see summary in Chiang \& Murray-Clay 2007) and references therein), and the discussion on how long accretion can be sustained once disks start to evolve is still open. The relatively large sample of evolved and transitional disks in $\sigma$ Ori is well suited to study the accretion properties of transition and evolved disks. However, the sample discussed in this paper includes too few such objects to provide an answer.

\subsection{Accretion evolution}

The relevance of the $\sigma$ Ori results for the understanding of the accretion evolution can be better understood in comparison with other regions of star formation, both younger and older.

The comparison with the $\rho$ Oph star forming region is particularly interesting. Mass accretion rates in $\rho$ Oph have been derived by Natta et al. (2006) for an IR-selected sample of Class II 


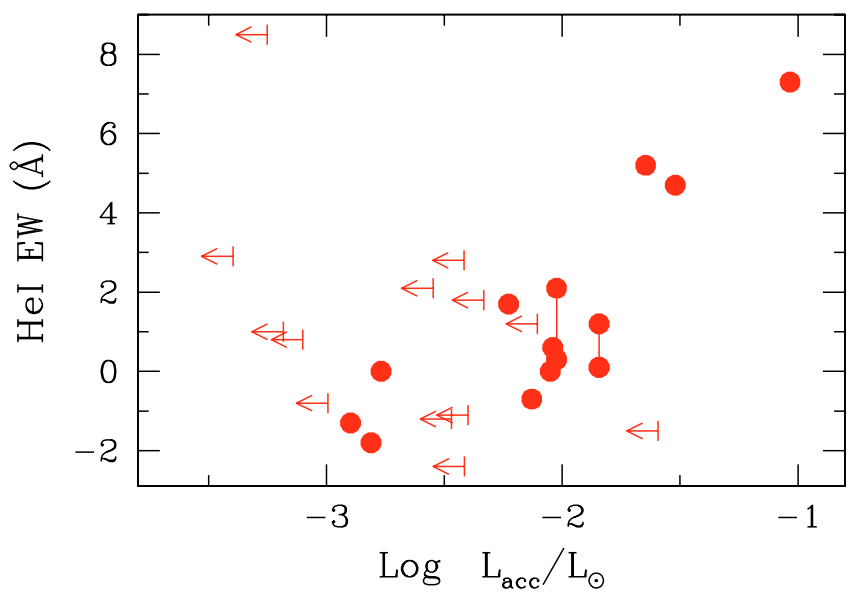

Fig. 8. HeI equivalent width as function of $L_{\text {acc }}$ for Class II objects. Dots are objects with an actual measurements of $L_{\text {acc }}$, while arrows are upper limits. For clarity, we do not plot the objects with $L_{\text {acc }}$ upper limits and non-detection of the He I line. For the two cases where we resolve the P-Cygni profile of the HeI line, we show (connected by a line) the corresponding unresolved equivalent width and the HeI activity index (sum of absolute values of absorption and emission equivalent widths; Edwards et al. 2006).

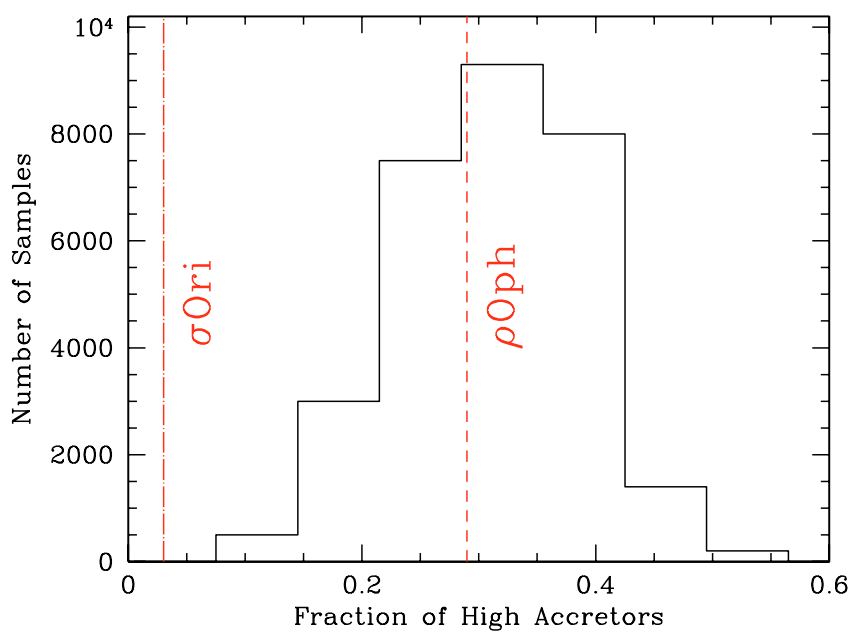

Fig. 9. Distribution of simulated fraction of high accretors in samples extracted from a parent distribution ias measured in $\rho$ Oph. The dashed line shows the fraction of high accretors in $\rho$ Oph, the dot-dashed line that in $\sigma$ Ori.

complete to a limiting mass of about $0.03 M_{\odot}$, using the luminosity of near-IR hydrogen recombination lines as a proxy of the accretion luminosity. Because the selection criteria and methods are similar, the comparison between these two regions is more meaningful than in other cases.

The major difference between the two regions is the lack in $\sigma$ Ori of objects with high accretion rates. If we restrict the comparison to the mass range $0.1-0.5 M_{\odot}$, where the two samples overlap, and take as a reference the line shown in Fig. 7, which is the best fit to the $\rho$ Oph sample of measured $\dot{M}_{\text {acc }}$ over the whole mass interval, we find that only 1 star in $\sigma$ Ori lies above this line (3\%), while the fraction is $\sim 30 \%$ in $\rho$ Oph.

Because the $\sigma$ Ori sample is small (much smaller than the $\rho$ Oph one), we performed a simplified Monte Carlo simulation to assess the statistical significance of this difference. The goal of our simulation is to evaluate whether the number of high accretors we observe in the $\sigma$ Ori sample is consistent with the distribution of rates derived from the $\rho$ Oph Class II objects

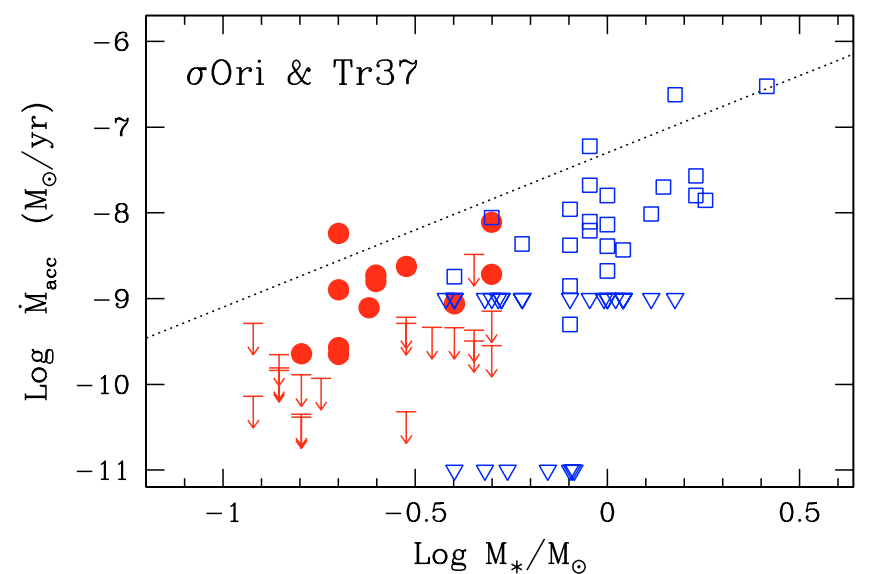

Fig. 10. $\dot{M}_{\text {acc }}$ vs. $M_{*}$ for $\sigma$ Ori (dots and arrows; same as Fig. 7) and Tr37 (from Sicilia-Aguilar et al. 2006b). Squares shows value of $\dot{M}_{\text {acc }}$ derived from $U$-band photometry, triangles at $\log \dot{M}_{\text {acc }}=-9 M_{\odot} /$ yr are objects with no $U$-band excess but broad $\mathrm{H} \alpha$, triangles at $\log \dot{M}_{\text {acc }}=-11$ are objects with no $U$-band excess and narrow $\mathrm{H} \alpha$. Note that for this group Sicilia-Aguilar et al. estimate $\log \dot{M}_{\text {acc }}<-12$, we plot them at -11 for convenience.

observations. We take the $\rho$ Oph stars in the interval 0.1-1 $M_{\odot}$ (the sample $0.1-0.5 M_{\odot}$ would have too few sources to derive a reliable $\dot{M}_{\text {acc }}$ distribution) and use the relationship between accretion rates and stellar mass derived by Natta et al. (2006) to scale all measurements to a fiducial stellar mass of $1 M_{\odot}$; we then compute the fraction of high accretors as the ratio of objects with measurements above the fiducial line, shown in Fig. 7, to the total (measurements plus upper limits). Within the chosen mass range, the fractions of high accretors in $\rho$ Oph and $\sigma$ Ori are $29 \%$ and $3 \%$, respectively.

Using a Monte Carlo method, we extract a large number of samples of 31 accretion rates. For each sample, we randomly remove a number of objects based on the fraction of upper limits in $\rho$ Oph $(\sim 52 \%)$, while for the remaining objects we simulate measurements using the distribution of measured rates. Figure 9 summarizes the results: the histogram plots the distribution of the fraction of high accretors in the simulations, the dashed line shows the value for the $\rho$ Oph sample, while the dashed-dotted line shows the value observed in our $\sigma$ Ori sample. The value observed in $\sigma$ Ori is lower than the 0.1 percentile of the Monte Carlo simulations, suggesting that it is very unlikely that the two samples are two different realizations of the same population.

The lack of strong accretors in $\sigma$ Ori is very likely an age effect, as in viscous disks, the mass accretion rate is expected to decrease with time (roughly as $t^{-1.5}$; Hartmann et al. 1998). On average, this means approximately a factor of 10 from the young (less than $1 \mathrm{Myr}) \rho$ Oph population to the older ( $3 \mathrm{Myr}$ ) $\sigma$ Ori one, consistent with the results shown in Fig. 7. One way of looking at this is to compute the fraction of high accretors in $\sigma$ Ori not with respect to the "younger" $\rho$ Oph $\dot{M}_{\text {acc }}-M_{*}$ fiducial line, but with respect to an "aged" version, where $\dot{M}_{\text {acc }}$ has decreased by the same factor of 10 for each $M_{*}$. Then, the fraction of $\sigma$ Ori high accretors will be $\sim 30 \%$, identical to the fraction of high accretors in $\rho$ Oph.

In Fig. 10 we show a comparison between mass accretion rates in $\sigma$ Ori and in the $\sim 4$ Myr old star forming region $\operatorname{Tr} 37$. This region, located at a distance of $\sim 900 \mathrm{pc}$, has been extensively studied by Sicilia-Aguilar et al. (2004, 2005, 2006a,b), who have classified disk properties from Spitzer IRAC and MIPS data and measured $\dot{M}_{\text {acc }}$ from $U$ band excess and $\mathrm{H} \alpha$ profiles. The methods of deriving $\dot{M}_{\text {acc }}$ are different in the two regions, 
but probably any systematic effect is within the uncertainties, since both correlations between the $U$-band excess and $L_{\text {acc }}$, and between the hydrogen IR line luminosity and $L_{\text {acc }}$, have been empirically calibrated using the same sample of TTS in Taurus with good measurements of $L_{\text {acc }}$ from veiling (e.g., Calvet and Gullbring 1998; Muzerolle et al. 1998).

In Fig. 10 we plot all the stars with disks (bona fide Class II and transition disks) with measured stellar mass and $\dot{M}_{\text {acc }}$, as reported by Siciliar-Aguilar et al. (2006b). We have not included any of the very young ( $\sim 1$ Myr) Class II objects, probably an episode of triggered star formation, found in the TR 37 "globule". Because of its larger distance, the Tr 37 sample covers a mass range between $\sim 0.4$ and $1.8 M_{\odot}$, with one star of $2.5 M_{\odot}$ (whose $\dot{M}_{\text {acc }}$ is very uncertain); there is practically no overlap with the $\sigma$ Ori lower-mass sample. However, the accretion properties of the two regions look very similar. The trend of higher $\dot{M}_{\text {acc }}$ for higher $M_{*}$ is confirmed, with good merging between the two samples. More importantly, both $\sigma$ Ori and Tr 37 lack high accretors to a similar degree. If we define, as before, high accretors to be those stars with values of $\dot{M}_{\text {acc }}$ above the fiducial line shown in Fig. 7, the fraction of high accretors in $\operatorname{Tr} 37$ is $6 \%$, which is similar, within the uncertainties, to the $3 \%$ found in $\sigma$ Ori, and certainly much lower than the $30 \%$ fraction of high accretors in $\rho$ Oph.

The continuity in the $\dot{M}_{\text {acc }}-M_{*}$ behavior between the two regions seems to extends to stars more massive than about 1$1.2 M_{\odot}$. In other star-forming regions, as discussed in Sect. 1, stars more massive than $\sim 1 M_{\odot}$ loose evidence of disks and accretion much faster than less massive stars, and one could expect to see a drop in the fraction of Class II stars among the more massive members of $\operatorname{Tr} 37$. However, this does not seem to be the case because $\operatorname{Tr} 37$ has a relatively high fraction of Class II (8 with respect to 5 Class III among stars more massive than $1.2 M_{\odot}$ ), even when the globule population is not taken into account. Of these, 6 have evidence of accretion. The case of $\operatorname{Tr} 37$ shows that the evolution of disks and accretion as a function of the stellar mass is still an open problem that needs to be addressed in more detail in the future.

\section{Summary and conclusions}

This paper presents $J$-band spectra of 35 stars in the star-forming region $\sigma$ Ori. We concentrate our attention on the three lines that are indicative of accretion-related activity, namely the two hydrogen recombination lines, $\mathrm{Pa} \beta$ and $\mathrm{Pa} \gamma$, and the $\mathrm{He} \mathrm{I}$ line at $1.083 \mu \mathrm{m}$.

The stars range in mass from $\sim 0.1$ to $\sim 0.5 M_{\odot}$. Four have no evidence of disks (Class III), according to the classification of Hernández et al. (2007) based on Spitzer near and mid-IR photometry. None of the three lines is detected in their spectra, with the possible exception of one star which has a tentative detection of He I emission. The other 31 objects have associated disks (Class II and evolved disks), and we detect Pa $\gamma$ in 12 of them and $\mathrm{Pa} \beta$ in 5 . The He I line is seen in 23 disk objects, either in emission or in absorption or both. Even at the low spectral resolution of our spectra $\left(\sim 300 \mathrm{~km} \mathrm{~s}^{-1}\right)$, in 2 cases the He I has a P-Cygni profile with redshifted absorption.

We derive accretion luminosities and mass accretion rates from the Pay luminosity; the results for $\sigma$ Ori indicate values much lower than in stars of similar mass in the younger regions Taurus and $\rho$ Oph. In particular, a statistical analysis shows that the $\rho$ Oph and $\sigma$ Ori populations cannot be drawn from the same parent population. TTS in $\sigma$ Ori are statistically identical to those in $\rho$ Oph only if we take into account their age, i.e., that any $\rho$ Oph object will have, at $\sigma$ Ori age, a value of $\dot{M}_{\text {acc }}$ lower by a factor of about $10\left(\dot{M}_{\text {acc }} \propto t^{-1.5}\right)$, as expected if viscosity controls disk evolution.

The HeI is detected (in net absorption or emission) in more stars than either of the hydrogen lines. Its behavior agrees qualitatively with what was found by Edwards et al. (2006) in a sample of TTS in Taurus; namely, strong HeI emission characterizes on average high accretors, while net absorption, probably due to a wind, is typical of low accretors. We extend this result to objects of very low $\dot{M}_{\text {acc }}$, and suggest that the He I line may be used to detect weak accretors when other methods become unfeasible.

The $\sigma$ Ori region, with its well-studied disk population and wealth of data at all available wavelengths, and with its large number of member stars extending over a broad mass range and moderate distance, is particularly well suited for a number of follow-up studies. Among them, we want to mention that it will be relatively easy to obtain near-IR spectra of the complete sample of Class II objects, down to the brown dwarf regime, to check, e.g., if the accretion rate evolves differently in brown dwarf disks. The comparison of $\sigma$ Ori with the region $\operatorname{Tr} 37$, which has similar age and a comparable fraction of high accretors, but a surprisingly high fraction of disks among stars more massive than about $1 M_{\odot}$, shows that the dependence of disk and accretion evolution with the mass of the central object is still an open and intriguing problem.

Our results on the two evolved/transition disks in our sample confirm that accretion is still going on in some of them and suggest that near-IR spectroscopy and high resolution spectra of the hydrogen and He I lines may help to clarify the last stages of the history of accretion disks.

Acknowledgements. We thank James Muzerolle for providing us with unpublished results of his magnetospheric accretion models and for very useful discussions. It is a pleasure to acknowledge the continuous, competent and friendly support of the ESO staff during the preparation and execution of the observations at La Silla observatory. This project was partially supported by MIUR grant 2004025227/2004 and by the INAF 2006 grant "From Disks to Planetary Systems".

\section{References}

Barrado y Navascués, D., \& Martín, E. L. 2003, AJ, 126, 2997

Bary, J. S., \& Matt, S. P. 2007, Star-disk Interaction in Young Star, ed. J. Bouvier, \& I. Appenzeller, IAU Symp., 243, in press

Béjar, V. J. S., Zapatero-Osorio, M. R., \& Rebolo, R. 1999, ApJ, 521, 671

Bessell, M. S. 1979, PASP, 91, 543

Bessell, M. S. 1991, AJ, 101, 662

Bessell, M. S., \& Brett, J. M. 1988, PASP, 100, 1134

Béjar, V. J. S., et al. 2001, ApJ, 556, 830

Brown, A. G. A., de Geus, E. J., \& de Zeeuw, P. T. 1994, A\&A, 289, 101

Caballero, J. A. 2007, A\&A, 466, 917

Caballero, J. A., Béjar, V. J. S., Rebolo, R., et al. 2007, A\&A, 470, 903

Calvet, N., \& Gullbring, E. 1998, ApJ, 509, 802

Calvet, N., Muzerolle, J., Briceño, C., Hernández, J., \& Hartmann, L. 2004, AJ, 128,1294

Carpenter, J. M., Mamajek, E. E., Hillenbrand, L. A., \& Meyer, M. R. 2006, ApJ, 651, L49

Chiang, E. I., \& Murray-Clay, R. A. 2007, Nat. Phys., 3, 604

Dahm, S. E., \& Hillenbrand, L. A. 2007, AJ, 133, 2072

D’Alessio, P., Calvet, N., Hartmann, L., Franco-Hernández, R., \& Servín, H. 2006, ApJ, 638, 314

D’Antona, F., \& Mazzitelli, I. 1997, Mem. S. A. It., 68, 807

Dullemond, C. P., \& Dominik, C. 2005, A\&A, 434, 971

Edwards, S., Fischer, W., Hillenbrand, L., \& Kean, J. 2006, ApJ, 646, 319

Franciosini, E., Pallavicini, R., \& Sanz-Forcada, J. 2006, A\&A, 446, 501

Folha, D. F. M., \& Emereso, J. P. 2001, A\&A, 365, 90

Hartmann, L., Calvet, N., Gullbring, E., \& D’Alessio, P. 1998, ApJ, 495, 385

Hernández, J., Hartmann, L., Megeath, T., et al. 2007, ApJ, 662, 1067

Hillenbrand, L. A., Strom, E. S., Calvet, N., et al. 1998, AJ, 116, 1816 
Hollenbach, D. J., Yorke, H. W., \& Johnstone, D. 2000, in Protostars and Planets IV, ed. V. Mannings, A. P. Boss, \& S. S. Russel (The University of Arizona Press), 410

Kenyon, S. J., \& Hartmann, L. 1995, ApJS, 101, 117

Kenyon, M. J., Jeffries, R. D., Naylor, T., Oliveira, J. M., \& Maxted, P. F. L. 2005, MNRAS, 356, 89

Lada, C. J., Muench, A. A., Luhman, K. L., et al. 2006, AJ, 131, 1574

Mohanty, S., Jayawardanha, J., \& Basri, G. 2005, ApJ, 626, 948

Muzerolle, J., Hartmann, L., \& Calvet, N. 1998, AJ, 116, 2965

Muzerolle, J., Calvet, N., \& Hartmann, L. 2001, ApJ, 550, 944

Muzerolle, J., Hillenbrand, L., Calvet, N., Bricen̈o, C., \& Hartmann, L. 2003, ApJ, 592, 266

Natta, A., Testi, L., Muzerolle, J., et al. 2004, A\&A, 424, 603

Natta, A., Testi, L., \& Randich, S., 2006, A\&A, 452, 245

Oliveira, J. M., Jeffries, R. D., \& van Loon, J. Th. 2004, MNRAS, 347, 1327
Oliveira, J. M., Jeffries, R. D., van Loon, J. Th., \& Rushton, M. T. 2006, MNRAS, 369, 272

Sacco, G., Franciosini, E., Randich, S. \& Pallavicini, R. 2007, A\&A, submitted Sherry, W. H., Walter, F. M., \& Wolk, S. J. 2004, AJ, 128, 2316

Sicilia-Aguilar, A., Hartmann, L. W., Bricen̈o, C., Muzerolle, J., \& Calvet, N. 2004, AJ, 128, 805

Sicilia-Aguilar, A., Hartmann, L. W., Hernández, J., Bricen̈o, C., \& Calvet, N. 2005, AJ, 130, 188

Sicilia-Aguilar, A., Hartmann, L. W., Calvet, N., et al. 2006a, ApJ, 638, 897

Sicilia-Aguilar, A., Hartmann, L. W., Fürész, G., et al. 2006b, AJ, 132, 2135

Skrutskie, M. F., Dutkevitch, D., Strom, S. E., et al. 1990, AJ, 99, 1187

Wolk, S. J. 1996, Ph.D. Thesis, State Univ. (New York: Stony Brook)

Zapatero Osorio, M. R., Béjar, V. J. S., Pavlenko, Ya., et al. 2002, A\&A, 384, 937 